9

\title{
Linear Interpolation and Estimation using Interval Analysis
}

\author{
S. M. Markov and E. D. Popova ${ }^{1}$
}

\begin{abstract}
This chapter considers interpolation and curve fitting using generalized polynomials under bounded measurement uncertainties from the point of view of the solution set (not the parameter set). It characterizes and presents the bounding functions for the solution set using interval arithmetic. Numerical algorithms with result verification and corresponding programs for the computation of the bounding functions in given domain are reported. Some examples are presented.
\end{abstract}

\subsection{INTRODUCTION: Formulation of the Problem}

We consider the problems of interpolation and curve fitting in the presence of unknown but bounded errors in the output measurements. Let $\eta\left(\lambda_{;} \cdot\right): D \rightarrow R$, $D \subseteq R^{k}$, be a model function depending on a real argument $\xi \in D$, and on a parameter vector $\lambda \in \Lambda \subset R^{m}$. The following hypotheses are assumed $[1,2,3,4]$ :

\footnotetext{
${ }^{1}$ S. M. Markov and E. D. Popova, present address: Institute of Mathematics \& Informatics, Bulgarian Academy of Sciences, Acad. G. Bonchev str., bl. 8, BG-1113 Sofia, Bulgaria, e-mail: \{smarkov, epopova\}@bio.bas.bg, fax: (+359 2) 9713649.

Reprint from: Bounding Approaches to System Identification, M. Milanese at al. Plenum Press, New York, Chap 9., 1996, pp. 139-157.
} 
Assumptions on the modeling function. The modeling function $\eta(\lambda ; \cdot)$ defined on some domain $D \subseteq R^{k}$ is a generalized polynomial depending linearly on $m$ parameters:

$$
\eta(\lambda ; \xi)=\sum_{i=1}^{m} \lambda_{i} \varphi_{i}(\xi)=\varphi(\xi)^{\top} \lambda, \quad \xi \in D,
$$

where $\varphi(\cdot)=\left(\varphi_{1}(\cdot), \ldots, \varphi_{m}(\cdot)\right)^{\top}$ is a vector of $m$ continuous on $D$ functions and $\lambda=$ $\left(\lambda_{1}, \ldots, \lambda_{m}\right)^{\top} \in R^{m}$ is a vector of $m$ (unknown) parameters. For any $\left(x_{1}^{\prime}, \ldots, x_{m}^{\prime}\right)$, $x_{i}^{\prime} \in D, i=1, \ldots, m$, the vector $\varphi(\cdot)$ generates a matrix defined by

$$
\left(\begin{array}{ccc}
\varphi_{1}\left(x_{1}^{\prime}\right) & \ldots & \varphi_{m}\left(x_{1}^{\prime}\right) \\
\vdots & \ddots & \vdots \\
\left.\varphi_{1}\left(x_{m}^{\prime}\right)\right) & \ldots & \varphi_{m}\left(x_{m}^{\prime}\right)
\end{array}\right)
$$

We shall assume that (2) is not singular whenever $\left(x_{1}^{\prime}, \ldots, x_{m}^{\prime}\right)$ is such that $x_{i}^{\prime} \neq x_{j}^{\prime}$, $i \neq j$. A set $\varphi$ of functions satisfying the above assumption will be further called a (Chebyshev) system of basic functions. The class of all modeling functions of the form (1) where $\varphi$ is a system of basic functions is denoted by $\mathcal{L}_{m}(D, \varphi)$ or $\mathcal{L}$.

Assumptions on the type of errors in the data. The input data are error-free and the output data errors are unknown but bounded (UBB) [5], [6]. This means that there are $n$ distinct (input) data $x_{j} \in D \subseteq R^{k}, j \in J=\{1, \ldots, n\}$, and there are $n$ (output) interval measurements $Y_{j}=\left[y_{j}^{-}, y_{j}^{+}\right], j \in J$, which contain the correct values of the corresponding measured quantities.

Denote the system of input data by $\mathbf{x}=\left(x_{1}, x_{2}, \ldots, x_{n}\right)^{\top} \in R^{n \times k}$ and the system of output measurements by $Y=\left(Y_{1}, \ldots, Y_{n}\right)^{\top} \in I R^{n}$, where $I R^{n}$ is the set of all $n$-dimensional interval vectors [7], [8] (see also [9]). Geometrically, the pairs $\left(x_{j}, Y_{j}\right), j \in J$, can be considered as $n$ vertical segments in the $(k+1)$-dimensional space $O x_{1} x_{2} \ldots x_{k} y$.

Throughout the paper it is assumed that $m \leq n$. In section 2 we consider the problem of finding bounds for the set of modeling functions $\eta \in \mathcal{L}_{m}(D)$ interpolating the vertical segments $\left(x_{j}, Y_{j}\right), j \in J$. More precisely, for a fixed $\xi \in D$ we look for the set of values at $\xi$ of all modeling functions $\eta$ interpolating the segments $\left(x_{j}, Y_{j}\right), j \in J$, that is in the set:

$$
\left\{\eta(\lambda ; \xi) \mid \eta \text { is such that } \eta\left(\lambda ; x_{j}\right) \in Y_{j}, j \in J\right\}, \quad \xi \in D .
$$

The requirement that the values of $\eta$ at $x_{j}$ range in the corresponding intervals $Y_{j}$ leads to a system of inequalities for $\lambda$

$$
\eta\left(\lambda ; x_{j}\right)=\varphi\left(x_{j}\right)^{\top} \lambda \in Y_{j}, \quad j \in J,
$$

which can be written in matrix form as 


$$
\Phi(\mathbf{x}) \lambda \in Y,
$$

where $\Phi(\mathbf{x})$ is the following $(n \times m)$-matrix of full $\operatorname{rank} \Phi(\mathbf{x})=m$ :

$$
\Phi(\mathbf{x})=\left(\begin{array}{ccc}
\varphi_{1}\left(x_{1}\right) & \ldots & \varphi_{m}\left(x_{1}\right) \\
\vdots & \ddots & \vdots \\
\left.\varphi_{1}\left(x_{n}\right)\right) & \ldots & \varphi_{m}\left(x_{n}\right)
\end{array}\right)=\left(\begin{array}{c}
\varphi\left(x_{1}\right)^{\top} \\
\vdots \\
\varphi\left(x_{n}\right)^{\top}
\end{array}\right) .
$$

In (4) the data $\mathbf{x}$ and $Y$ are known, the parameter $\lambda$ is unknown. We thus have to solve a system of $n$ algebraic inclusions for the $m$-dimensional parameter $\lambda$. Any $\lambda$ satisfying (4) is called a feasible parameter. Every feasible parameter $\lambda$ generates a solution function $\eta(\lambda ; \cdot) \in \mathcal{L}_{m}(D)$. Denote by $\Lambda$ the set of all feasible parameters, and by $\eta(\Lambda ; \xi)$ the set of values of all solution functions at $\xi \in D$, respectively

$$
\begin{gathered}
\Lambda=\left\{\lambda \in R^{m} \mid \Phi(\mathbf{x}) \lambda \in Y\right\}, \\
\eta(\Lambda ; \xi)=\left\{\varphi(\xi)^{\top} \lambda \mid \lambda \in \Lambda\right\}, \quad \xi \in D .
\end{gathered}
$$

The set $\eta(\Lambda ; \xi)$ defined by ( 7$)$ is an interval for any fixed $\xi \in D$. Thus (7) defines an interval-valued function (briefly, interval function) on $D$, which will be further denoted by $\eta(\mathbf{x}, Y ; \cdot)$. Note the difference between $\eta(\Lambda ; \cdot)=\left\{\varphi(\cdot)^{\top} \lambda \mid \lambda \in \Lambda\right\}$ and $\eta(\mathbf{x}, Y ; \cdot)$ : the former is a set of solution functions defined on $D$ (sometimes called feasible solution set), whereas the latter is an interval function defined on $D$. Of course for a fixed $\xi \in D$ we have $\eta(\Lambda ; \xi)=\eta(\mathbf{x}, Y ; \xi)$. We shall be particularly concerned with characterizing and computing the bounding lower and upper functions $\eta^{-}(\mathbf{x}, Y ; \cdot), \eta^{+}(\mathbf{x}, Y ; \cdot)$ of the interval function $\eta(\mathbf{x}, Y ; \cdot)$, which are called enveloping functions for the feasible solution set $\eta(\Lambda ; \cdot)[10]$.

We can compute $\eta(\mathbf{x}, Y ; \xi)$ for $\xi \in D$ by solving two constrained linear optimization problems [4], [6]

$$
\eta(\mathbf{x}, Y ; \xi)=\left[\min _{\lambda \in \Lambda}\left\{\varphi(\xi)^{\top} \lambda\right\}, \max _{\lambda \in \Lambda}\left\{\varphi(\xi)^{\top} \lambda\right\}\right] .
$$

Another approach is to enclose $\Lambda$ by an interval vector (box) $\Lambda^{I}[3]$, and then find an enclosure for $\eta(\mathbf{x}, Y ; \xi)$ by $\eta(\mathbf{x}, Y ; \xi) \subseteq \varphi(\xi)^{\top} \Lambda^{I}$.

The problem of finding/enclosing the interval function $\eta(\mathbf{x}, Y ; \cdot)$ is quite different from the problem of finding/enclosing the parameter set $\Lambda$ defined by (6) (see $[2,3,5,6,11])$. The set $\Lambda$ is an $m$-dimensional polytope, whereas $\eta(\mathbf{x}, Y ; \xi)$ is a closed one-dimensional interval for a fixed $\xi$. The presentation or computation of $\eta(\mathbf{x}, Y ; \xi)$ in a given domain for $\xi$ can be of practical importance. In the case of one-dimensional argument $\xi$ we characterize the interval function $\eta(\mathbf{x}, Y ; \cdot)$ and propose methods for its presentation and computation. A computer program written in PASCAL-SC [12] is reported, which efficiently computes the interval function $\eta(\mathbf{x}, Y ; \xi)$ in a given interval. 
If the interpolation problem has no solution, then we often want to solve it by choosing another family of modeling functions (e. g. by changing either the number of parameters or the system of basic functions). We may choose to reformulate the interpolation problem as a curve fitting (estimation) problem [5], [6]. We assume that the inclusions (4) can be violated, which practically means that the errors in the measurements are assumed to be of a stochastic nature.

In Section 3 we consider the problem of finding the set of parameters $\lambda$, respectively the set of modeling functions $\eta(\lambda ; \cdot)$, such that

$$
\eta\left(\lambda ; x_{j}\right) \approx Y_{j}, j \in J
$$

in matrix form $\Phi(\mathbf{x}) \lambda \approx Y$, where the symbol $\approx$ means that the values $\eta\left(\lambda ; x_{i}\right)=$ $\varphi\left(x_{j}\right)^{\top} \lambda$ are "close" to the measurement intervals $Y_{j}$. For the numerical (singlevalued) case $Y=y \in R^{n}$ the curve fitting problem (9) is mathematically formulated by choosing an operator (called estimator) $\phi(y)$ producing from a data set $(\mathbf{x}, y)$ a solution function $\eta\left(\lambda_{y} ; \cdot\right)$ from $\mathcal{L}_{m}(X)$. The operator $\phi$ is chosen in accordance with the hypothesis on the statistical nature of the errors in the measurements (for instance, least-square estimator is chosen if the errors in $y$ are assumed to have normal distribution). Let us restrict ourselves to so-called projection estimators ([1],[5]) of the form $\phi(y)=\eta\left(\lambda_{y} ; \cdot\right)$, with $\lambda_{y}$ minimizing some functional of the form

$$
\left\|y-\Phi(\mathbf{x}) \lambda_{y}\right\|=\inf _{\lambda \in K}\|y-\Phi(\mathbf{x}) \lambda\|, \quad K \subseteq R^{m}
$$

where $\|\cdot\|$ is a norm in $R^{n}$. Assume as before that the measurement interval $Y$ contains the true values of the measured quantities. As proposed in $[1,5,6]$ we consider the set of solution functions corresponding to the data $(\mathbf{x}, Y)$, defined by

$$
\left\{\eta\left(\lambda_{y} ; \cdot\right) \mid y \in Y\right\}=\left\{\varphi(\cdot)^{\top} \lambda_{y} \mid y \in Y\right\}
$$

where $\lambda_{y}$ is given in (10).

Let $\Lambda_{\phi}$ be the set of all $\lambda_{y}$, produced by the estimator $\phi(y)$, whenever the numerical vector $y$ ranges in the interval measurement vector $Y=\left(Y_{1}, \ldots, Y_{n}\right)$,

$$
\Lambda_{\phi}=\left\{\lambda_{y} \in K \subseteq R^{m}, \lambda_{y} \text { satisfies (10) } \mid y \in Y\right\} .
$$

The set $\Lambda_{\phi}$ is called the estimate uncertainty set [5], [6]. The set $\Lambda_{\phi}$ generates a corresponding (estimate) solution set

$$
\eta\left(\Lambda_{\phi} ; \cdot\right)=\left\{\varphi(\cdot)^{\top} \lambda \mid \lambda \in \Lambda_{\phi}\right\} .
$$

For a fixed $\xi \in D$ we have

$$
\eta\left(\Lambda_{\phi} ; \xi\right)=\left\{\varphi(\xi)^{\top} \lambda \mid \lambda \in \Lambda_{\phi}\right\}, \quad \xi \in D .
$$


Equality (14) defines an interval-valued function; section 3 is devoted to its presentation and computation.

This chapter considers the interval-valued functions generated by the solution sets both for the interpolation and for the curve fitting problems. In some special cases we find subregions of $D$ where the interval solution functions have simple presentation and can be easily computed. Next subsection gives a brief introduction to the necessary concepts of interval arithmetic.

\subsection{Interval Arithmetic: Basic Concepts}

By $I R$ we denote the set of all intervals $Y$ of the form $Y=\left[y^{-}, y^{+}\right]=\left\{y \mid y^{-} \leq\right.$ $\left.y \leq y^{+}\right\}, y^{-}, y^{+} \in R$. Throughout the paper we shall use two simple interval arithmetic operations [7], [8]: one for addition of two intervals $X, Y \in I R$ and one for multiplication by a real number $\alpha \in R$ defined as follows:

$$
\begin{aligned}
X+Y & =\left[x^{-}+y^{-}, x^{+}+y^{+}\right], \\
\alpha X & =\left[\alpha x^{-\sigma(\alpha)}, \alpha x^{\sigma(\alpha)}\right]= \begin{cases}{\left[\alpha x^{-}, \alpha x^{+}\right],} & \alpha \geq 0, \\
{\left[\alpha x^{+}, \alpha x^{-}\right],} & \alpha<0 .\end{cases}
\end{aligned}
$$

wherein $\sigma(\alpha)=\{-, \alpha<0 ;+, \alpha \geq 0\}, x^{--}=x^{+}, x^{-+}=x^{-}$.

We shall make use of the following simple application of interval arithmetic. Given a real valued vector $\alpha=\left(\alpha_{1}, \ldots, \alpha_{n}\right)$ and an interval valued vector $Y=$ $\left(Y_{1}, \ldots, Y_{n}\right)^{\top}$ we can write

$$
\begin{aligned}
& \{\alpha y \mid y \in Y\} \\
= & \left\{\alpha_{1} y_{1}+\alpha_{2} y_{2}+\ldots+\alpha_{n} y_{n} \mid y_{1} \in Y_{1}, \ldots, y_{n} \in Y_{n}\right\} \\
= & \alpha_{1} Y_{1}+\alpha_{2} Y_{2}+\ldots+\alpha_{n} Y_{n}=\alpha Y .
\end{aligned}
$$

A standard way to present the set $\{\alpha y \mid y \in Y\}$ via the end-points of $Y$ is

$$
\{\alpha y \mid y \in Y\}=\left[\sum_{i=1}^{n} \alpha_{i} y_{i}^{-\sigma\left(\alpha_{i}\right)}, \sum_{i=1}^{n} \alpha_{i} y_{i}^{\sigma\left(\alpha_{i}\right)}\right] .
$$

The interval expression (15) is much shorter than expression (16), which does not make use of interval arithmetic.

Remark. A similar expression to (16) can be obtained by using a presentation of the intervals via centers and radii (see e. g. [6], Proposition 1). Denoting the center of the interval $Y_{i}$ by $y_{i}^{c}$ and its radius by $y_{i}^{r}$ we obtain the expression

$$
\{\alpha y \mid y \in Y\}=\left[\sum_{i=1}^{n} \alpha_{i}\left(y_{i}^{c}-\sigma\left(\alpha_{i}\right) y_{i}^{r}\right), \sum_{i=1}^{n} \alpha_{i}\left(y_{i}^{c}+\sigma\left(\alpha_{i}\right) y_{i}^{r}\right)\right],
$$

which is also clumsy, whereas the interval expression $\alpha Y$ is very brief and offers many convenience, as we shall further see. 
More generally, if $A$ is a real valued $(k \times n)$-matrix

$$
A=\left(\begin{array}{cccc}
a_{11}, & a_{12}, & \ldots, & a_{1 n} \\
& & \ldots & \\
a_{k 1}, & a_{k 2}, & \ldots, & a_{k n}
\end{array}\right)=\left(\begin{array}{c}
a_{1} \\
\vdots \\
a_{k}
\end{array}\right)
$$

then using (15) we obtain for the $k$-dimensional set $\{A y \mid y \in Y\}$ the following inclusion

$$
\begin{aligned}
\{A y \mid y \in Y\} & =\left\{\left(a_{1} y, a_{2} y, \ldots, a_{k} y\right) \mid y \in Y\right\} \\
& \subseteq\left(a_{1} Y, \ldots, a_{k} Y\right)=A Y
\end{aligned}
$$

The inclusion (17) is often known as "wrapping effect" [8]. The set $A Y$ is the smallest $k$-dimensional box (orthotope, interval vector) enclosing the set $\{A y \mid y \in$ $Y\}$.

\subsection{LINEAR INTERPOLATION UNDER INTERVAL MEASUREMENTS}

\subsubsection{The Multidimensional Case}

First consider the general situation $k \geq 1, D \subseteq R^{k}$ and the problem of finding the interpolation interval function (8).

Definition 9.1 For a fixed class $\mathcal{L}=\mathcal{L}_{m}(D, \varphi)$ of modeling functions a system of vertical segments $(\mathbf{x}, \tilde{Y}), \mathbf{x}=\left(x_{1}, \ldots, x_{n}\right)^{\top}, \tilde{Y}=\left(\tilde{Y}_{1}, \ldots, \tilde{Y}_{n}\right)^{\top}$, is called $\mathcal{L}-$ compatible (or just compatible), if for any $i \in J=\{1, \ldots, n\}$ and $y_{i} \in \tilde{Y}_{i}$ there is an element $\eta$ of $\mathcal{L}$, with $\eta\left(x_{i}\right)=y_{i}$, such that $\eta\left(\lambda ; x_{j}\right) \in \tilde{Y}_{j}$ for $j=1, \ldots, n, j \neq i$.

In the situation when the data matrix $\mathbf{x}$ is fixed (as is the case in this paper) we shall sometimes say " $\tilde{Y}$ is $\mathcal{L}$-compatible", instead of " $(\mathbf{x}, \tilde{Y})$ is $\mathcal{L}$-compatible".

Denote $\tilde{Y}=\left(\tilde{Y}_{1}, \tilde{Y}_{2}, \ldots, \tilde{Y}_{n}\right)^{\top}, \tilde{Y}_{i}=\eta\left(\mathbf{x}, Y ; x_{i}\right), i=1, \ldots, n$. Then $\tilde{Y}_{i}=$ $\eta\left(\mathbf{x}, \tilde{Y} ; x_{i}\right)$ that is, the interval vectors $\tilde{Y}$ and $Y$ generate same feasible solution sets. The compatible segments $\left(x_{i}, \tilde{Y}_{i}\right), i \in J$, have the property of possessing no "excess points", that is such points, for which no individual solution function $\eta$ passes through [13].

Two systems $(\mathbf{x}, Y),(\mathbf{x}, \tilde{Y})$, generating same feasible solution sets are called equivalent. The problem of finding a solution set corresponding to the data $(\mathbf{x}, Y)$, can be divided into two steps: 1$)$ to find an $\mathcal{L}$-compatible system $(\mathbf{x}, \tilde{Y})$ which is equivalent to $(\mathbf{x}, Y)$, and 2) to find the solution set generated by $(\mathbf{x}, \tilde{Y})$.

Every feasible parameter $\lambda \in \Lambda$ generates a vector $y_{\lambda}=\left(y_{1}, \ldots, y_{n}\right)^{\top} \in R^{n}$ by

$$
y_{j}=\eta\left(\lambda ; x_{j}\right), \quad j \in J
$$

in matrix form $y_{\lambda}=\Phi(\mathbf{x}) \lambda$. The set of all vectors $y$ defined by (18) for some $\lambda \in \Lambda$ will be denoted 


$$
\begin{aligned}
Y^{\prime} & =\left\{y_{\lambda}=\left(\eta\left(\lambda ; x_{1}\right), \ldots, \eta\left(\lambda ; x_{n}\right)\right)^{\top} \mid \lambda \in \Lambda\right\} \\
& =\{\Phi(\mathbf{x}) \lambda \mid \lambda \in \Lambda\} .
\end{aligned}
$$

In other words $Y^{\prime}$ is the subset of all $y, y \in Y$, for which the system $y=\Phi(\mathbf{x}) \lambda$ is consistent. For a compatible set of data $(\mathbf{x}, Y)$ the interval $Y_{j}$ is the projection of the set $Y^{\prime}$ defined by (19) on the $j$-th coordinate axis.

Let us first consider the case $n=m$ when the number of data equals the number of parameters. In this case $Y^{\prime}=Y$ ( since $y=\Phi(\mathbf{x}) \lambda$ is consistent for all $y \in Y$ ) and we can express the solution set by means of the following proposition.

Proposition 9.1 For $m=n$ we have

$$
\eta(\mathbf{x}, Y ; \xi)=\left(\varphi(\xi)^{\top} \Phi^{-1}(\mathbf{x})\right) Y
$$

Proof. A modeling function $\eta(\lambda ; \cdot)=\varphi(\cdot)^{\top} \lambda$ from $\mathcal{L}_{m}(X)$, which interpolates a set of $m$ data $(\mathbf{x}, Y)$, satisfies a system $\Phi(\mathbf{x}) \lambda \in Y$ of $m$ algebraic inclusions for the $m$ unknown parameters, or $\Phi(\mathbf{x}) \lambda=y, y \in Y$. For $n=m$ we have $Y^{\prime}=Y$. Since $\operatorname{det} \Phi(\mathbf{x}) \neq 0$, every $y \in Y$ generates a $\lambda=\Phi^{-1}(\mathbf{x}) y$. For the set of values of the modeling function interpolating $(\mathbf{x}, Y)$ at a fixed $\xi \in D$ we obtain respectively

$$
\begin{aligned}
\eta(\mathbf{x}, Y ; \xi)=\left\{\varphi(\xi)^{\top} \lambda \mid \lambda \in \Lambda\right\} & =\left\{\varphi(\xi)^{\top}\left(\Phi^{-1}(\mathbf{x}) y\right) \mid y \in Y\right\} \\
& =\left\{\left(\varphi(\xi)^{\top} \Phi^{-1}(\mathbf{x})\right) y \mid y \in Y\right\} \\
& =\left(\varphi(\xi)^{\top} \Phi^{-1}(\mathbf{x})\right) Y .
\end{aligned}
$$

The interval function (20) will be further called simple interval interpolation function (SII-function).

Remark. Proposition 1 shows that the SII-function can be computed for every $\xi$ in interval arithmetic using the simple interval-arithmetic expression (20). In (20) the vector $\varphi(\xi)^{\top} \Phi^{-1}(\mathbf{x})$ is multiplied by the interval vector $Y$ in the sense of (15). Such an exact presentation can not be given for the parameter set $\Lambda$ because of the wrapping effect [8]. Indeed for the set $\Lambda$ of feasible parameters we obtain

$$
\Lambda=\left\{\Phi^{-1}(\mathbf{x}) y \mid y \in Y^{\prime}\right\}=\left\{\Phi^{-1}(\mathbf{x}) y \mid y \in Y\right\} \subseteq \Phi^{-1}(\mathbf{x}) Y .
$$

Using interval arithmetic we thus obtain the inclusion $\Lambda \subseteq \Phi^{-1}(\mathbf{x}) Y=\Lambda^{I}$, which may be rough. This is due to the fact that $\Lambda$ is a convex polytope, whereas $Y$, resp. $\Lambda^{I}$, is a $m$-dimensional box (see [8]). The above consideration also demonstrate the importance of the brackets in (20). A change of the place of the brackets leads to an inclusion

$$
\left(\varphi(\xi)^{\top} \Phi^{-1}(\mathbf{x})\right) Y \subseteq \varphi(\xi)^{\top}\left(\Phi^{-1}(\mathbf{x}) Y\right)
$$


Indeed $\Lambda \subseteq \Phi^{-1}(\mathbf{x}) Y=\Lambda$ implies

$$
\begin{aligned}
\eta(\mathbf{x}, Y ; \xi) & =\left\{\varphi(\xi)^{\top} \lambda \mid \lambda \in \Lambda\right\} \subseteq\left\{\varphi(\xi)^{\top} \lambda \mid \lambda \in \Lambda^{I}\right\} \\
& =\varphi(\xi)^{\top}\left(\Phi^{-1}(\mathbf{x}) Y\right) .
\end{aligned}
$$

We now consider the case $m<n$. In this case $Y^{\prime} \subseteq Y$ and the inclusion $\Lambda \subseteq \Lambda^{I}$ due to

$$
\Lambda=\left\{\Phi^{-1}(\mathbf{x}) y \mid y \in Y^{\prime}\right\} \subseteq\left\{\Phi^{-1}(\mathbf{x}) y \mid y \in Y\right\} \subseteq \Phi^{-1}(\mathbf{x}) Y=\Lambda^{I}
$$

can be very rough. The following proposition gives a characterization of the solution set $\eta(\mathbf{x}, Y ; \cdot)$ (see Lemma 2 from $[6])$.

Proposition 9.2 There exists a subset $Q$ of the index set $J=\{1, \ldots, n\}$ consisting of $m$ elements $(Q \subseteq J, \operatorname{card}(Q)=m)$, such that for every $l \in Q$ at least one of the two equalities $\eta^{-}\left(\mathbf{x}, Y ; x_{l}\right)=Y_{l}^{-}, \eta^{+}\left(\mathbf{x}, Y ; x_{l}\right)=Y_{l}^{+}$hold.

The proof of this Proposition is given in [6]. Proposition 2 shows that the solution set reaches the end-points of at least $m$ input intervals $Y_{l}, l \in Q \subseteq J$.

Let the index set $Q$ be a subset of the index set $J$ with $m$ elements: $Q \subseteq J$, $\operatorname{card}(Q)=m$. Assume that $Q$ is ordered in increasing order and let $q(i)$ be the $i$-th element of $Q$. Denote by $\mathbf{x}^{Q}=\left(x_{q(1)}, \ldots, x_{q(m)}\right)^{\top}$ the matrix $\mathbf{x}$ reduced to the index set $Q$. Analogously $Y^{Q}=\left(Y_{q(1)}, \ldots, Y_{q(m)}\right)^{\top}$ is the vector $Y$ reduced to $Q$.

To find the set of functions from $\mathcal{L}_{m}(X)$ interpolating a reduced set of $m$ data $\left(\mathbf{x}^{Q}, Y^{Q}\right)$ we consider the corresponding system $\Phi\left(\mathbf{x}^{Q}\right) \lambda \in Y^{Q}$, which is a system of $m$ algebraic inclusions for $m$ unknown parameters and applying (20), obtain $\eta\left(\mathbf{x}^{Q}, Y^{Q} ; \xi\right)=\left(\varphi(\xi)^{\top} \Phi^{-1}\left(\mathbf{x}^{Q}\right)\right) Y^{Q}$.

Proposition 9.3 The value of $\eta(\mathbf{x}, Y ; \cdot)$ at a point $\xi$ is given by

$$
\eta(\mathbf{x}, Y ; \xi)=\bigcap_{Q \subseteq J} \eta\left(\mathbf{x}^{Q}, Y^{Q} ; \xi\right)=\bigcap_{Q \subseteq J}\left(\varphi(\xi)^{\top} \Phi^{-1}\left(\mathbf{x}^{Q}\right)\right) Y^{Q} .
$$

The proof is obvious. Proposition 3 shows that the value of $\eta(\mathbf{x}, Y ; \cdot)$ at $\xi$ can be determined by an intersection of $\left(\begin{array}{c}n \\ m\end{array}\right)$ SII-functions.

The intervals $Y_{j}$ can be reduced to $\mathcal{L}$-compatible intervals $\tilde{Y}_{j}$ using the following

Proposition 9.4 For the $\mathcal{L}$-compatible intervals we have

$$
\tilde{Y}_{j}=Y_{j} \cap \bigcap_{Q \subseteq J} \eta\left(x^{Q}, Y^{Q} ; x_{j}\right) .
$$


We suggest the following methods for the computation of $\eta(\mathbf{x}, Y ; \cdot)$ at a point $\xi \in D:$

A. Compute $\eta(\mathbf{x}, Y ; \cdot)$ at $\xi$ by means of Proposition 3 , that is by intersecting the values of all simple interval interpolating functions at $\xi$. The latter are computed by means of Proposition 1. If at some point $\xi$ the interval values of two simple interval interpolating functions are disjointed, their intersection is an empty set and the set of solution functions is void.

B. For $\xi \in D$ compute $\eta(\mathbf{x}, Y ; \xi)$ by solving two constrained linear optimization problems (8).

C. Compute first the $\mathcal{L}$-compatible intervals $\tilde{Y}_{i}$ by means of Proposition 4 . Then compute $\eta(\mathbf{x}, Y ; \cdot)=\eta(\mathbf{x}, \tilde{Y} ; \cdot)$ at arbitrary $\xi$ by using method $\mathbf{A}$ or $\mathbf{B}$ for the compatible intervals.

Below we look for effective methods for the presentation and computation of $\eta$ in the one-dimensional case $k=1$.

\subsubsection{The One-Dimensional Case}

In the remaining part of this section we shall assume $k=1$, that is the input data $\mathbf{x}$ is a vector of real components and will be denoted by $x=\left(x_{1}, \ldots, x_{n}\right)$. We shall assume that the components of $x$ belong to an interval $X=\left[x^{-}, x^{+}\right]$and that $x_{0}=x^{-} \leq x_{1}<x_{2}<\ldots<x_{n} \leq x^{+}=x_{n+1}$. We shall use the letter $k$ to denote a fixed subinterval $\left[x_{k}, x_{k+1}\right]$ to be considered. The following theorem gives an additional characterization of the boundary functions of the solution set.

We first give a definition which will be used in the proof of the next proposition.

Definition 9.2 (see [11], [14]) For $l \leq m$ a l-face of $\Lambda$ is a subset of $\Lambda$ defined by

$$
y_{j}^{-} \leq \varphi\left(\xi_{j}\right)^{\top} \lambda \leq y_{j}^{+}, \quad j \in J,
$$

where $m-l$ of the above linear independent inequalities transform into equalities.

Proposition 9.5 (see [15], [16], [17]) Let the set $\eta(\lambda ; \cdot)$ of all functions from $\mathcal{L}_{m}(X)$ which interpolate $(x, Y)$ be not empty and let the interval function $\eta(x, Y ; \cdot)$ be the envelope of this set. Then in every $\left(x_{k}, x_{k+1}\right), k=0,1, \ldots, n$, the upper and lower boundary functions of $\eta(x, Y ; \cdot)$ are functions from $\mathcal{L}_{m}(X)$ generated by some parameters $\lambda_{k}^{-}, \lambda_{k}^{+} \in \Lambda$.

Proof. Proposition 9.5 states that for every subinterval $\left[x_{k}, x_{k+1}\right]$ there exist two parameters $\lambda_{k}^{-}, \lambda_{k}^{+} \in \Lambda \subseteq R^{m}$ generating the envelope in the whole subinterval, that is

$$
\begin{array}{ll}
\eta^{-}(x, Y ; \xi)=\eta\left(\lambda_{k}^{-} ; \xi\right)=\varphi(\xi)^{\top} \lambda_{k}^{-}, & \xi \in\left(x_{k}, x_{k+1}\right) \\
\eta^{+}(x, Y ; \xi)=\eta\left(\lambda_{k}^{+} ; \xi\right)=\varphi(\xi)^{\top} \lambda_{k}^{+}, & \xi \in\left(x_{k}, x_{k+1}\right) .
\end{array}
$$


Assuming the opposite, there exist a point $\xi_{s} \in\left(x_{k}, x_{k+1}\right)$ and two parameters $\lambda^{1}, \lambda^{2} \in R^{m}, \lambda^{1} \neq \lambda^{2}$, such that $\eta^{+}\left(x, Y ; \xi_{s}\right)=\varphi\left(\xi_{s}\right)^{\top} \lambda^{1}=\varphi\left(\xi_{s}\right)^{\top} \lambda^{2}$. On the other hand we have

$$
\eta^{+}\left(x, Y ; \xi_{s}\right)=\max _{\lambda \in \Lambda} \varphi\left(\xi_{s}\right)^{\top} \lambda .
$$

Because the set of optimal points of the linear programming problem (22) is convex all points of the segment $\left[\lambda^{1}, \lambda^{2}\right]$ are optimal. The set of all optimal points of $(22)$ is a $l$-face of $\Lambda$, where $l \geq 1$ and the vector $\varphi\left(\xi_{s}\right)$ is perpendicular to this $l$-face of $\Lambda$. The $l$-face is an intersection of $(m-l)$ hyperplanes with linear independent normal vectors $a^{1}, \ldots, a^{m-l} \in\left\{\varphi\left(\xi_{j}\right), j \in J\right\}$ (the linear independence follows from the assumption that the modeling function is from $\left.\mathcal{L}_{m}(X)\right)$. Thus the vector $\varphi\left(\xi_{s}\right)$ is a linear combination of $a^{1}, \ldots, a^{m-l}$. This is a contradiction to the assertion that $\varphi$ is a system of basic functions. For the lower function $\eta^{-}$the arguments are analogous.

Proposition 9.5 shows, that under the given assumptions the upper and lower boundary functions $\eta(x, Y ; \xi)$ for all $\xi \in\left(x_{k}, x_{k+1}\right)$ are themselves elements of $\mathcal{L}_{m}(X)$, and, therefore, to find $\eta(x, Y ; \xi)$ for $\xi \in\left[x_{k}, x_{k+1}\right]$ we have to determine expressions for these two functions. Such expressions can be found ether in terms of some subset $\left(x^{Q}, Y^{Q}\right)$ of the given data or in terms of $\lambda$ depending on the method used: intersection of $S I I$-functions (method $\mathbf{A}$ ) or constrained optimization (method B).

In some cases it can be preferable to use method $\mathbf{C}$ which prescribes first the computation of the compatible intervals. The next proposition shows that, if the set of data $(x, Y)$ is $\mathcal{L}$-compatible, then $\eta(x, Y ; \xi)$ may be determined by an intersection of a reduced number of simple interval interpolating functions.

Proposition 9.6 If the set of data $(x, Y)$ is $\mathcal{L}$-compatible, then for every $k=$ $0, \ldots, n$ the following formula holds

$$
\eta(x, Y ; \xi)=\bigcap_{Q \in Q(k)} \eta\left(x^{Q}, Y^{Q} ; \xi\right) \text { for } \xi \in\left[x_{k}, x_{k+1}\right]
$$

where $Q(k)$ is the set of all subsets $Q$ of $J$ consisting of $m$ elements (notationally, $Q \subseteq J, \operatorname{card}(Q)=m)$, such that

$$
\begin{aligned}
k, k+1 \in Q, & \text { if } \quad 0<k<n, \\
1, n \in Q, & \text { if } \quad k=0 \text { or } k=n .
\end{aligned}
$$

If $m=2$ the set $Q(k)$, for every $k, 0 \leq k \leq n$, consist of one single pair, namely

$$
Q(k)= \begin{cases}\{k, k+1\}, & \text { if } 0<k<n, \\ \{1, n\}, & \text { if } k=0 \text { or } k=n .\end{cases}
$$


For the interval solution $\eta(\lambda ; \cdot)$ in this case $(m=2$, compatible data) the following simple formula holds in $\left[x_{k}, x_{k+1}\right]$ :

$$
\begin{aligned}
\eta(x, Y ; \xi) & =\eta\left(x^{Q(k)}, Y^{Q(k)} ; \xi\right)=\left(\varphi(\xi)^{\top} \Phi^{-1}\left(\mathbf{x}^{Q(k)}\right)\right) Y^{Q(k)} \\
& = \begin{cases}\frac{\Delta\left(\xi, x_{k+1}\right)}{\Delta\left(x_{k}, x_{k+1}\right)} Y_{k}+\frac{\Delta\left(x_{k}, \xi\right)}{\Delta\left(x_{k}, x_{k+1}\right)} Y_{k+1}, & \text { if } 0<k<n, \\
\frac{\Delta\left(\xi, x_{n}\right)}{\Delta\left(x_{1}, x_{n}\right)} Y_{1}+\frac{\Delta\left(x_{1}, \xi\right)}{\Delta\left(x_{1}, x_{n}\right)} Y_{n}, & \text { if } k=0 \text { or } k=n,\end{cases}
\end{aligned}
$$

wherein $\Delta\left(x^{\prime}, x^{\prime \prime}\right)=\left|\begin{array}{ll}\varphi_{1}\left(x^{\prime}\right) & \varphi_{2}\left(x^{\prime}\right) \\ \varphi_{1}\left(x^{\prime \prime}\right) & \varphi_{2}\left(x^{\prime \prime}\right)\end{array}\right|$.

Clearly, finding the compatible intervals in the case $m=2$ solves the problem of finding the $S I I$-function, which is then obtained by connecting the upper, resp., lower end-points of each two neighboring segments $\left(x_{i}, Y_{i}\right),\left(x_{i+1}, Y_{i+1}\right)$ via generalized linear functions. Proposition 6 is proved (for the polynomial case) in $[16]$.

Numerical algorithm (for $k=1$ ). Compute $\eta(x, Y ; \cdot)$ at some point $\xi_{i}$ from the open interval $\left(x_{i}, x_{i+1}\right)$, e.g. $\xi_{i}=\left(x_{i+1}+x_{i}\right) / 2$, using method $\mathbf{A}$ or B. Proposition 9.5 states that there are two unique generalized polynomials $\eta_{i}^{-}=$ $\eta\left(\lambda_{i}^{-} ; \xi\right), \eta_{i}^{+}=\eta\left(\lambda_{i}^{+} ; \xi\right)$ which are the boundary functions of $\eta(x, Y ; \cdot)$ in the interval $\left[x_{i}, x_{i+1}\right]$. We can find expressions for the boundary functions $\eta_{i}^{-}, \eta_{i}^{+}$by any one of the methods $\mathbf{A}$ or $\mathbf{B}$. Using method $\mathbf{A}$ we obtain two $m$-dimensional subsets $Q_{i}^{-}$, $Q_{i}^{+}$of $J$ and two $m$-dimensional sets of binary variables $\mathcal{A}^{-}=\left(\alpha_{q(1)}^{-}, \ldots, \alpha_{q(m)}^{-}\right), \mathcal{A}^{+}$ $=\left(\alpha_{q(1)}^{+}, \ldots, \alpha_{q(m)}^{+}\right), \alpha_{q(i)}^{-}, \alpha_{q(i)}^{+} \in\{+,-\}, i=1, \ldots, n$, such that for $\xi \in\left[x_{i}, x_{i+1}\right]$ :

$$
\begin{aligned}
& \eta^{-}(x, Y ; \xi)=\left(\varphi(\xi)^{\top} \Phi^{-1}\left(\mathbf{x}^{Q_{i}^{-}}\right)\right)\left(Y^{Q_{i}^{-}}\right)^{\alpha_{q(i)}^{-}} \\
& \eta^{+}(x, Y ; \xi)=\left(\varphi(\xi)^{\top} \Phi^{-1}\left(\mathbf{x}^{Q_{i}^{+}}\right)\right)\left(Y^{Q_{i}^{+}}\right)^{\alpha_{q(i)}^{+}}
\end{aligned}
$$

(note that the pairs $\left(Q_{i}^{-}, \mathcal{A}_{i}^{-}\right),\left(Q_{i}^{+}, \mathcal{A}_{i}^{+}\right)$may not be unique, and any pair can be used).

Alternatively, if method $\mathbf{B}$ is used then we can determine $\lambda_{i}^{-}, \lambda_{i}^{+}$as defined by Proposition 9.5.

According to Proposition 9.5 the expressions for the functions $\eta_{i}^{-}, \eta_{i}^{+}$can be used for presentation or computation of $\eta(x, Y ; \cdot)$ at any point in the subinterval $\left[x_{i}, x_{i+1}\right]$.

\subsubsection{The Polynomial Case}

If the basic functions are of the form $\varphi_{i}(x)=x^{i-1}, \quad i=1, \ldots, m$, then $(2)$ is the Vandermond's determinant: $\operatorname{det} \Phi\left(x^{\prime}\right)=\prod_{i>j}\left(x_{i}-x_{j}\right)$, which does not vanish. $\mathcal{L}_{m}(X)$ is the class of polynomial functions defined on $X=R$ of $(m-1)$-st degree of the form $\eta_{m-1}(\lambda ; \xi)=\lambda_{1}+\lambda_{2} \xi+\ldots+\lambda_{m} \xi^{m-1}$. 
In the case $n=m$ formula (20) for the $S I I$-function obtains the form

$$
\eta_{m-1}(x, Y ; \xi)=l(x ; \xi)^{\top} Y, \quad l_{i}(x ; \xi)=\prod_{k=1, \ldots, m, k \neq i} \frac{\xi-x_{k}}{x_{i}-x_{k}},
$$

(see [18], [19]). This interval function has been studied (without using interval arithmetic) in [20].

Formula (21) for $n>m$ in the polynomial case reads [13]:

$$
\eta_{m-1}(x, Y ; \xi)=\bigcap_{Q \subseteq J} l\left(x^{Q} ; \xi\right)^{\top} Y^{Q}, \quad l_{i}\left(x^{Q} ; \xi\right)=\prod_{k=1, \ldots, m, k \neq i} \frac{\xi-x_{q(k)}}{x_{q(i)}-x_{q(k)}},
$$

wherein $Q=\{q(k)\}_{k=1}^{m}$.

The intervals $Y_{j}$ can be reduced to compatible intervals $\tilde{Y}_{j}$ by [13]

$$
\tilde{Y}_{j}=Y_{j} \cap \bigcap_{Q \subseteq J, j \notin Q} l\left(x^{Q} ; x_{j}\right)^{\top} Y^{Q}, \quad j \in J .
$$

For $m=2$ applying Proposition 6 we obtain for $\xi \in\left[x_{k}, x_{k+1}\right], 0 \leq k \leq n$, the following simple expression [13]

$$
\eta_{1}(x, Y ; \xi)= \begin{cases}\frac{\xi-x_{k+1}}{x_{k}-x_{k+1}} Y_{k}+\frac{\xi-x_{k}}{x_{k+1}-x_{k}} Y_{k+1}, & \text { if } 0<k<n, \\ \frac{\xi-x_{n}}{x_{1}-x_{n}} Y_{1}+\frac{\xi-x_{1}}{x_{n}-x_{1}} Y_{n}, & \text { if } k=0 \text { or } k=n,\end{cases}
$$

where the data $(x, Y)$ are assumed compatible.

Next are two examples for polynomial functions. The computations are performed by a program written in PASCAL-SC [12], based on the method $\mathbf{A}$.

Example 1. Let the following set of data be given

$$
(x, Y)=\left(\begin{array}{llll}
1, & 2, & 4, & 6 \\
{[1,3],} & {[1,2],} & {[1.5,2.5],} & {[2,3]}
\end{array}\right)^{\top},
$$

and let the modeling functions be second order polynomials of the form

$$
\eta_{2}(\lambda ; \xi)=\lambda_{1}+\lambda_{2} \xi+\lambda_{3} \xi^{2}
$$

The graph of the interval function $\eta_{2}(x, Y ; \cdot)$ is presented on Fig. 1. For comparison the simple interval polynomial $\eta_{3}(x, Y ; \cdot)$ is also presented. In order to recognize both interval interpolating functions on Fig. 1 we should keep in mind that $\eta_{2} \subseteq \eta_{3}$.

According to Proposition 3 the bounding functions of $\eta_{2}(x, Y ; \cdot)$ pass through at least three end-points of the interval segments, which fully determine them. The program gives results for $\eta_{2}(x, Y ; \cdot)$ presented in Table 1 . We see that the computed compatible intervals coincide with the input intervals, that is the input data are compatible. 


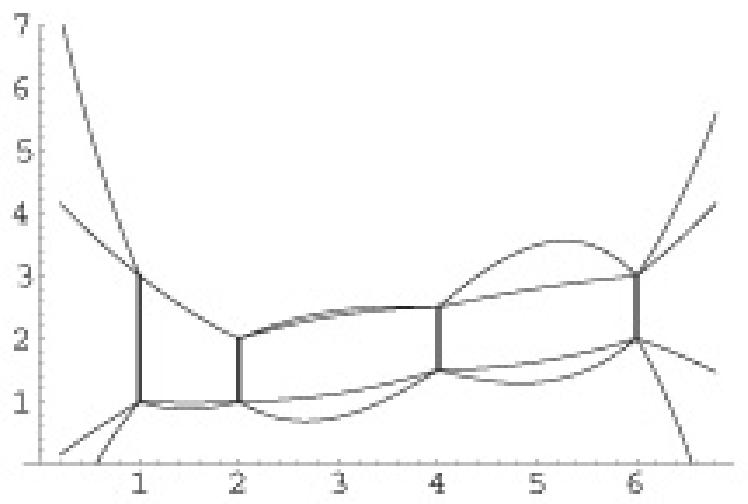

Figure 1: Graphs of the interval polynomials related to Example 1.

\begin{tabular}{cccc}
\hline \multirow{2}{*}{ Subinterval } & \multicolumn{2}{c}{ Bounding Functions } & \\
\cline { 2 - 3 } & Lower & Upper & Compatible Intervals \\
\hline \hline$\left[x_{-\infty}, x_{1}\right]$ & $Y_{1}^{-} Y_{3}^{+} Y_{4}^{-}$ & $Y_{2}^{+} Y_{3}^{-} Y_{4}^{+}$ & $Y_{1}=[1,3]$ \\
{$\left[x_{1}, x_{2}\right]$} & $Y_{1}^{-} Y_{2}^{-} Y_{4}^{+}$ & $Y_{2}^{+} Y_{3}^{-} Y_{4}^{+}$ & $Y_{2}=[1,2]$ \\
{$\left[x_{2}, x_{3}\right]$} & $Y_{2}^{-} Y_{3}^{-} Y_{4}^{+}$ & $Y_{2}^{+} Y_{3}^{+} Y_{4}^{-}$ & $Y_{3}=[1.5,2.5]$ \\
{$\left[x_{3}, x_{4}\right]$} & $Y_{2}^{+} Y_{3}^{-} Y_{4}^{-}$ & $Y_{1}^{-} Y_{3}^{+} Y_{4}^{+}$ & $Y_{4}=[2,3]$ \\
{$\left[x_{4}, x_{\infty}\right]$} & $Y_{1}^{-} Y_{3}^{+} Y_{4}^{-}$ & $Y_{2}^{+} Y_{3}^{-} Y_{4}^{+}$ & \\
\hline
\end{tabular}

Table 1: Bounding functions \& compatible intervals for the Problem of Example 1.

Remark. To demonstrate the advantages of our method of direct computation of the interval function $\eta_{2}(x, Y ; \cdot)$ let us compute the solution set for this example through the parameter set $\Lambda$. Assume that we have computed $\Lambda$ exactly. We then optimally enclose $\Lambda$ to obtain an interval vector $\Lambda^{I}$. Then the best result for the upper function is

$$
\eta_{2}^{+}\left(\Lambda^{I} ; \xi\right)=4.5+1.25 \xi+0.25 \xi^{2}
$$

and for the lower function

$$
\eta_{2}^{-}\left(\Lambda^{I} ; \xi\right)=-0.1-1.75 \xi-0.15 \xi^{2} .
$$

The width of $\eta_{2}\left(\Lambda^{I} ; \xi\right)$ at $\xi=6$ is $\omega\left(\eta_{2}\left(\Lambda^{I} ; 6\right)\right)=\eta_{2}^{+}\left(\Lambda^{I} ; 6\right)-\eta_{2}^{-}\left(\Lambda^{I} ; 6\right)=37$, whereas the width of $\eta_{2}(\Lambda ; 6)=\eta_{2}(x, Y ; 6)$ as computed by our method is

$$
\eta_{2}^{+}(x, Y ; 6)-\eta_{2}^{-}(x, Y ; 6)=1 .
$$




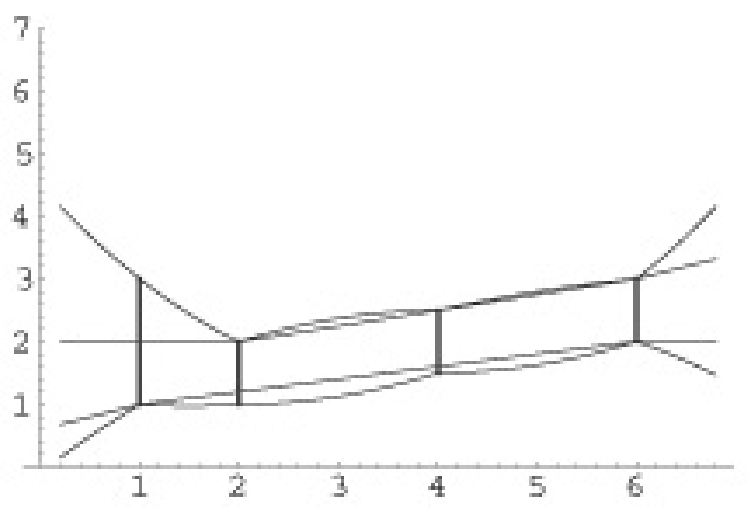

Figure 2: Graphs of the interval polynomials related to Example 2.

Example 2. For the same set of data and for the set of linear modeling functions $\eta_{1}(\lambda ; \xi)=\lambda_{1}+\lambda_{2} \xi$ we obtain the results presented in Table 2 .

\begin{tabular}{cccc}
\hline & \multicolumn{2}{c}{ Bounding Functions } & \\
\cline { 2 - 3 } Subinterval & Lower & Upper & Compatible Intervals \\
\hline \hline$\left[x_{-\infty}, x_{1}\right]$ & $Y_{1}^{-} Y_{4}^{+}$ & $Y_{2}^{+} Y_{4}^{-}$ & $Y_{1}=[1,2]$ \\
{$\left[x_{1}, x_{2}\right]$} & $Y_{1}^{-} Y_{4}^{-}$ & $Y_{2}^{+} Y_{4}^{-}$ & $Y_{2}=[1.2,2]$ \\
{$\left[x_{2}, x_{3}\right]$} & $Y_{1}^{-} Y_{4}^{-}$ & $Y_{2}^{+} Y_{4}^{+}$ & $Y_{3}=[1.6,2.5]$ \\
{$\left[x_{3}, x_{4}\right]$} & $Y_{1}^{-} Y_{4}^{-}$ & $Y_{3}^{+} Y_{4}^{+}$ & $Y_{4}=[2,3]$ \\
{$\left[x_{4}, x_{\infty}\right]$} & $Y_{2}^{+} Y_{4}^{-}$ & $Y_{1}^{-} Y_{4}^{+}$ & \\
\hline
\end{tabular}

Table 2: Bounding functions \& compatible intervals for the Problem of Example 2.

The interval function $\eta_{1}(x, Y ; \cdot)$, comprising the set of linear modeling functions is presented on Fig. 2. For comparison the function $\eta_{2}(x, Y ; \cdot)$ is given (the latter also appears in Fig. 1). To recognize both functions on Fig. 2 recall that $\eta_{1} \subseteq \eta_{2}$.

Example 3. We next consider an example using 6 knots $(x, Y)=\left(\begin{array}{cccccc}0 & 1 & 2 & 3 & 4 & 5 \\ {[1,1.02]} & {[0.99,1.25]} & {[1.04,1.06]} & {[1.07,1.09]} & {[1.16,1.18]} & {[1.23,1.25]}\end{array}\right)^{\top}$

Fig. 3 presents the corresponding polynomials $\eta_{5}(x, Y ; \cdot)$ and $\eta_{4}(x, Y ; \cdot)$. Of 


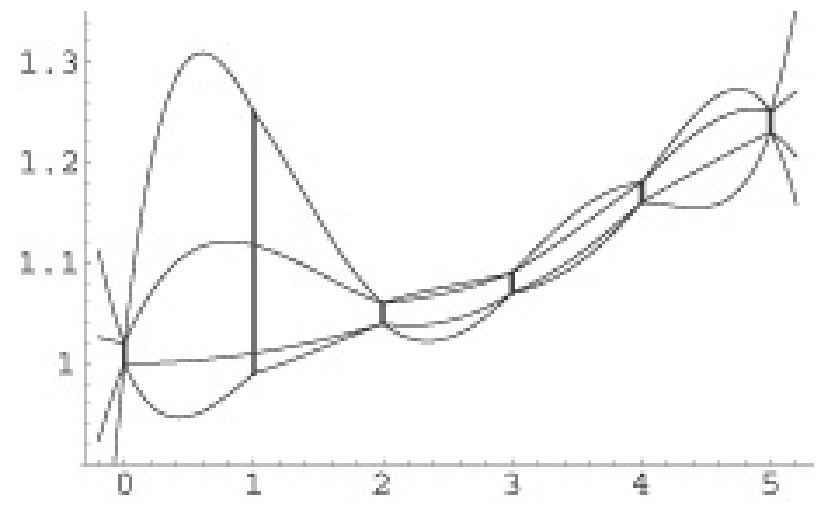

Figure 3: Graphs of the interval polynomials related to Example 3.

course, we have $\eta_{4} \subseteq \eta_{5}$.

\subsection{LINEAR ESTIMATION UNDER INTERVAL MEASUREMENTS}

In this section we shall assume that the parameter $\lambda_{y}$ defined by (10) depends linearly on $y$, i.e. $\lambda_{y}=H y$, where $H \in R^{m \times n}$, and $H=H(\mathbf{x})$ may depend on $\mathbf{x}$ but not on $y$, then formula (12) can be written as

$$
\begin{aligned}
\Lambda_{\phi} & =\left\{\lambda_{y} \in K \subseteq R^{m}, \lambda_{y}=H y \mid y \in Y\right\} \\
& =\{H y \mid y \in Y\} \subseteq H Y
\end{aligned}
$$

where by the last inclusion relation (17) has been used.

Let us assume as before that $\mathcal{L}$ is a class of linear on $\lambda$ functions of the form $\eta(\lambda ; \cdot)=\varphi(\cdot)^{\top} \lambda$ defined on $D$. For a fixed $\xi \in D$ the estimate solution set can be written in the form

$$
\begin{aligned}
\eta\left(\Lambda_{\phi} ; \xi\right) & =\left\{\eta(\lambda ; \xi) \mid \lambda \in \Lambda_{\phi}\right\} \\
& =\left\{\varphi(\xi)^{\top} \lambda, \lambda=H y \mid y \in Y\right\} \\
& =\left\{\varphi(\xi)^{\top}(H y) \mid y \in Y\right\}=\left\{\left(\varphi(\xi)^{\top} H\right) y \mid y \in Y\right\} \\
& =\left(\varphi(\xi)^{\top} H\right) Y=\Gamma(\xi) Y
\end{aligned}
$$

Note that the interval-valued function (24) gives the exact bounds for the solution set. We next consider a special case of least-square estimator illustrating the above approach.

Multiple linear regression. In the case of multiple linear regression, let us denote $\xi=\left(1, \xi_{1}, \ldots, \xi_{m-1}\right)$ and assume $\varphi_{i}(\xi)=\xi_{i}, i=0, \ldots, m-1$, so that 


$$
\begin{gathered}
\eta(\lambda ; \xi)=\varphi(\xi)^{\top} \lambda=\lambda_{0}+\lambda_{1} \xi_{1}+\ldots+\lambda_{m-1} \xi_{m-1}=\xi \lambda \text {. Denoting } \\
X=\left(\begin{array}{rrrr}
1 & x_{11} & \ldots & x_{1 m-1} \\
& & \ldots & \\
1 & x_{n 1} & \ldots & x_{n m-1}
\end{array}\right)
\end{gathered}
$$

we obtain from (10) with an $l_{2}$ norm the matrix $H$ in the form $H=\left(X^{\top} X\right)^{-1} X^{\top}$. Substituting in (23) and (24) we obtain

$$
\begin{aligned}
\Lambda_{\phi} & \subseteq H Y=\left(\left(X^{\top} X\right)^{-1} X^{\top}\right) Y, \\
\eta\left(\Lambda_{\phi} ; \xi\right) & =\Gamma(\xi) Y=(\xi H) Y=\left(\xi\left(X^{\top} X\right)^{-1} X^{\top}\right) Y,
\end{aligned}
$$

where $\Gamma(\xi)=\xi\left(X^{\top} X\right)^{-1} X^{\top}=\left(\gamma_{1}(\xi), \ldots, \gamma_{n}(\xi)\right)$.

In the case $m=2$, the approximating function is linear of the form $f(\lambda ; \xi)=$ $\lambda_{0}+\lambda_{1} \xi$. For the components $\gamma_{i}(\xi)$ of the $n$-dimensional vector $\Gamma(\xi)$ we obtain

$$
\begin{aligned}
\gamma_{i}(\xi) & =\left(\xi\left(X^{\top} X\right)^{-1} X^{\top}\right)_{i} \\
& =\left(x_{i}-\bar{x}\right)(\xi-\bar{x}) / S_{x x}+1 / n, i=1, \ldots, n,
\end{aligned}
$$

where $\bar{x}=\sum_{i=1}^{n} x_{i} / n, \quad S_{x x}=\sum_{i=1}^{n} x_{i}^{2}-n \bar{x}=\sum_{i=1}^{n}\left(x_{i}-\bar{x}\right)^{2}$. The boundary functions of the interval function $L(\xi)=\Gamma(\xi) Y$ are lines in each interval with endpoints two neighboring $\xi_{i}, \quad i=1, \ldots, n$, where $\xi_{i}$ are defined by $\gamma_{i}\left(\xi_{i}\right)=0$, that is (see also [21]):

$$
\xi_{i}=\bar{x}+S_{x x} /\left(n\left(\bar{x}-x_{i}\right)\right) .
$$

The polynomial and multinomial cases produce similar results under the corresponding choice of the matrix $X$.

Example 4. Consider the data

$$
(x, Y)=\left(\begin{array}{ll}
0, & {[1,1.02]} \\
1, & {[0.99,1.01]} \\
2, & {[1.04,1.06]} \\
3, & {[1.07,1.09]} \\
4, & {[1.16,1.18]} \\
5, & {[1.23,1.25]}
\end{array}\right) .
$$

For the given data, the set of interpolating polynomials of degree $m-1=2$ consists of only one single-valued interpolation polynomial. The latter serves also for an unique solution of the same problem with $m-1=3$ and $m-1=4$ (Fig. 4 ). The solution set is empty for the same problem with $m-1<2$. The envelope of the set of least-square approximation polynomials of second degree for the given interval data is also presented on Figure 4.

Example 5. For the set of data of Example 1 and for modeling functions which are second order polynomials the corresponding sets of solutions both for the interpolation and the least-square approximation problems are presented on Fig. 5. 


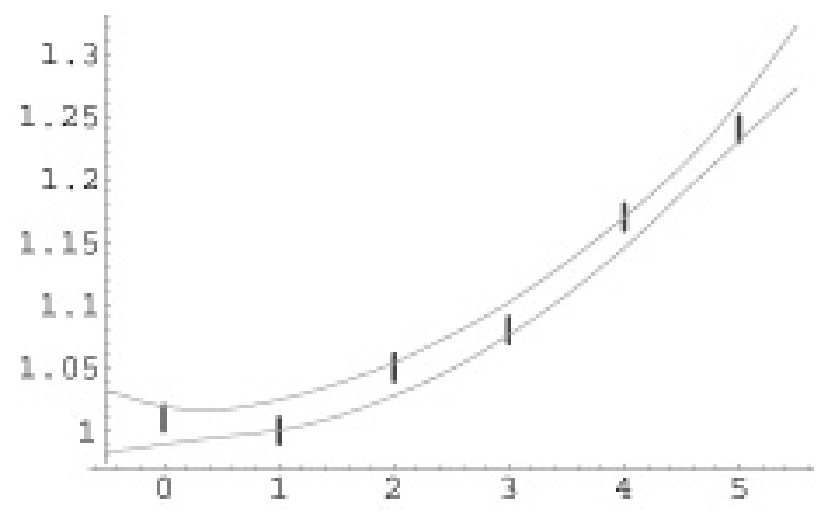

Figure 4: Graphs of the interval polynomials related to Example 4.

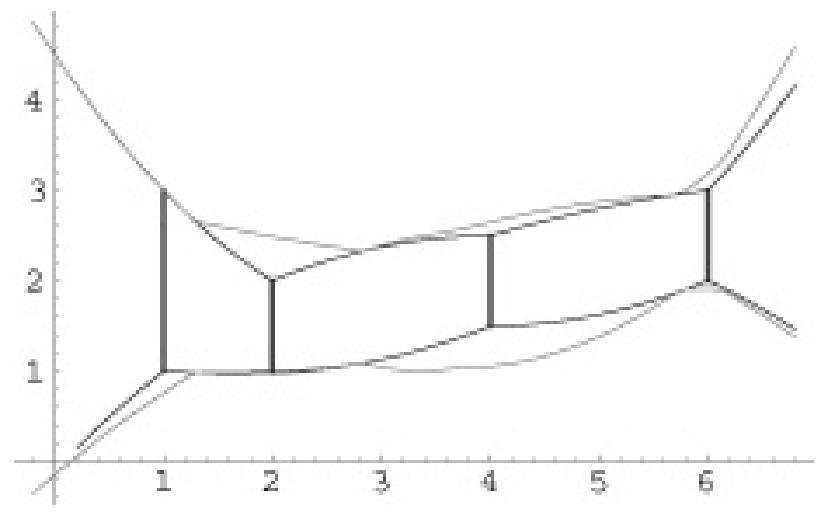

Figure 5: Graphs of the interval polynomials related to Example 5. 


\subsection{CONCLUSION}

We studied both interpolation and curve fitting problems involving generalized polynomials and interval data. In certain special cases we obtain exact intervalarithmetic expressions for the envelopes of the sets of solution functions (see expressions $(20),(21),(24),(26))$. In the one-dimensional case when the solutions are functions of one variable, we characterize the enveloping functions showing that they are piece-wise generalized polynomials. These interval-arithmetic expressions can be effectively computed in a software environment which supports interval arithmetic like recently developed SC-languages [12] (or computer algebra systems Maple and Mathematica). Such an environment provides computer operations with directed roundings, so that the computed interval bounds are automatically rounded towards outside and contain with guarantee the true results. Thus, the computed bounds comprise all possible kinds of input and computational errors. This fact opens a new way to the practical implementation and interpretation of the computed results especially with respect to the interpolation problem. For example, assume that we know that the experimentally obtained measurement intervals $Y$ contain with guarantee the true values of the measured quantities and that $\eta(x, Y ; \cdot)$ is the interval solution function computed from these measurements under the assumption that the model function $\eta$ belongs to $\mathcal{L}$. Assume that an experiment provides us with a new measurement $\left(x_{N}, Y_{N}\right)$ such that $\eta\left(x, Y ; x_{N}\right) \cap Y_{N}=\emptyset$. The correct conclusion then is that the class $\mathcal{L}$ of model functions is inadequate for the description of the experimental data.

Therefore our approach and programing tools can be used from experimental scientists, for checking hypotheses with respect to the type of the modeling functions. New data can be easily checked whether they intersect the available interval solution sets. If some of these intersections are empty then it follows that the type of the modeling functions is wrong. Then another type of modeling function (possibly involving more parameters or other type of basic functions) should be taken in consideration.

In the above arguments it is assumed that $Y_{i}$ are measurement intervals, containing with guarantee the true values of the measured quantities. It seems that experimental scientists can provide such intervals in most situations. Moreover, the provision of guaranteed bounds seems to be a substantial part of the experiment. At present experimental scientists often do not care about obtaining such bounds, which diminishes the value of the experiment. A possible explanation for such attitude is that still few mathematical tools and methods dealing with interval problems have been developed. Measurement tools and instruments also sometimes fail to provide the necessary guaranteed bounds for the data to be read off. The guaranteed numerical "interval approaches" should be employed for guaranteed interval data, possibly obtained using high quality "interval measurement" tools. 
ACKNOWLEDGEMENTS. This work has been partially supported by the Bulgarian National Foundation for Scientific Research under contract No MM10/91.

\section{References}

[1] M. Milanese and A. Vicino, in: Bounding Approaches to System Identification (M. Milanese at al., eds.), Plenum Press, New York, Chap. 21996.

[2] J. P. Norton, Automatica 23, 497 (1987).

[3] E. Walter (Ed.), Math. Comput. Simulation 32, 447 (1990).

[4] A. P. Voschinin and G. R. Sotirov, Optimization with Uncertainties, Moscow Energy Institute, Moscow, in Russian (1989).

[5] M. Milanese, in: Robustness in Identification and Control (M. Milanese, R. Tempo and A. Vicino, Eds.), pp. 3-24, Plenum Press, New York (1989).

[6] M. Milanese, G. Belforte, IEEE Trans. Autom. Control AC-27, 408 (1982).

[7] G. Alefeld, J. Herzberger, Introduction to Interval Computations, Academic Press, New York (1983).

[8] R. E. Moore, Interval Analysis, Prentice Hall, Englewood Cliffs, N. J. (1966).

[9] L. Jaulin and E. Walter, in: Bounding Approaches to System Identification (M. Milanese at al., eds.), Plenum Press, New York, Chap. 231996.

[10] G. Sotirov, in: Scientific Computation and Mathematical Modelling (S. M. Markov, Ed.), DATECS Publishing, Sofia, pp. 31-36 (1993).

[11] S. H. Mo, J. P. Norton, in: Proceedings of the 12th IMACS World Congress, Paris (1988).

[12] U. Kulisch (ed.), PASCAL-SC: A PASCAL-Extension for Scientific Computation. Information Manual and Disks for IBM PC, Wiley-Teubner, Chichester (1987).

[13] S. M. Markov, in: Computer Arithmetic, Scientific Computation and Mathematical Modeling (E. Kaucher, S. M. Markov, G. Mayer, Eds.), IMACS, pp. 251-262 (1991).

[14] S. N. Tschernikow, Lineare Ungleichungen, Deucher Verlag der Wissenschaften Berlin, Germany (1971).

[15] S. Markov, E. Popova, U. Schneider, J. Schulze, Mathematics and Computers in Simulation 42, pp. 35-45 (1993).

[16] U. Schneider, Modellierung unscharfer Daten - Intervallinterpolationsproblem, Diplomawork TH Merseburg, Fachbereich Mathematik und Informatik, Merseburg, Germany (1992).

[17] U. Schneider, J. Schulze, in: Scientific Computation and Mathematical Modelling (S. M. Markov, Ed.), DATECS Publishing, Sofia, pp. 173-176 (1993).

[18] J. Herzberger, SIAM J. Appl. Math. 34, 4 (1978).

[19] J. Garloff, Z. Angew. Math. Mech. 59, T59-T60 (1979).

[20] M. A. Crane, SIAM J. Appl. Math. 29, 4 (1975).

[21] S. M. Markov, in: Contributions to Computer Arithmetic and Self-Validating Numerical Methods (Ch. Ullrich, Ed.) J. C. Baltzer AG, Switzerland, pp. 133-147 (1990). 